\title{
All-optical clock recovery of NRZ-DPSK signals using optical resonator-type filters
}

\author{
Peucheret, Christophe; Seoane, Jorge; Ji, Hua
}

Published in:

Proceedings, OECC

Link to article, DOI:

10.1109/OECC.2009.5214507

Publication date:

2009

Document Version

Publisher's PDF, also known as Version of record

Link back to DTU Orbit

Citation (APA):

Peucheret, C., Seoane, J., \& Ji, H. (2009). All-optical clock recovery of NRZ-DPSK signals using optical resonator-type filters. In Proceedings, OECC (pp. 1-2). IEEE. https://doi.org/10.1109/OECC.2009.5214507

\section{General rights}

Copyright and moral rights for the publications made accessible in the public portal are retained by the authors and/or other copyright owners and it is a condition of accessing publications that users recognise and abide by the legal requirements associated with these rights.

- Users may download and print one copy of any publication from the public portal for the purpose of private study or research.

- You may not further distribute the material or use it for any profit-making activity or commercial gain

- You may freely distribute the URL identifying the publication in the public portal

If you believe that this document breaches copyright please contact us providing details, and we will remove access to the work immediately and investigate your claim. 


\title{
All-Optical Clock Recovery of NRZ-DPSK Signals Using Optical Resonator-Type Filters
}

\author{
Christophe Peucheret, Jorge Seoane and Hua Ji \\ Department of Photonics Engineering, Technical University of Denmark, DK-2800 Kgs. Lyngby, Denmark \\ Phone: +(45)45253840, Fax: +(45)45936581, Email: cpeu@fotonik.dtu.dk
}

\begin{abstract}
It is shown how introducing a limited rise time to the driving signal enables all-optical clock recovery of NRZDPSK signals generated using a phase modulator. A Fabry-Perot filter is used to generate the optical clock.
\end{abstract}

\section{Introduction}

Clock recovery of non return-to-zero differential phaseshift keying (NRZ-DPSK) signals is known to present some inherent difficulty due to the absence of a clock tone in the signal spectrum. This is especially critical when the NRZ-DPSK signal is generated using a phase modulator. Phase-to-intensity modulation (PM-IM) conversion, for instance in a delay interferometer, followed by detection and the use of conventional electrical clock recovery circuits is an obvious solution. However, at bit rates for which such circuits are not readily available and for the implementation of advanced all-optical processing techniques, the generation of an optical clock signal is a desirable feature. Two main approaches have been followed so far. The first one utilises an optical filter to perform some degree of PMIM conversion, followed by an all-optical clock recovery scheme such as a Fabry-Perot cavity [1] or a modelocked fibre ring laser [2]. The second approach uses clock enhancement via propagation of the NRZ-DPSK signal in a dispersive element, followed by injection of the signal in a mode-locked fibre ring laser for optical clock generation [3].

In this paper, it is shown numerically that introducing a finite rise time to the electrical signal driving the phase modulator in an NRZ-DPSK transmitter results in the presence of clock components in the signal spectrum that allow all-optical clock recovery by the mean of optical resonator type filters.

\section{Proof of principle}

The concept is illustrated in Fig. 1. A conventional NRZDPSK transmitter made from a continuous wave (CW) laser and a phase modulator (PM) is employed. The electrical signal is first differentially encoded before being applied to the PM with a peak-to-peak voltage ideally equal to the half-wave voltage of the modulator. An electrical low-pass filter (LPF) is used to model the rise time of the driving signal. In practice, this bandwidth limitation may also arise from the PM itself. The phase modulated signal is then input to an optical resonator filter, typically a Fabry-Perot (FP) cavity, with a free-spectral range (FSR) equal to the bit rate, a specified finesse $F$, and proper frequency tuning.

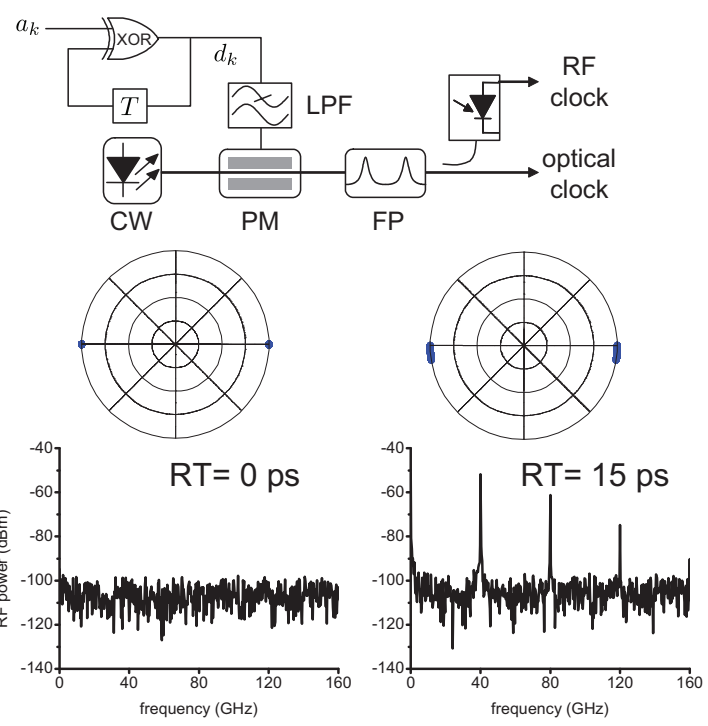

Fig. 1: Concept of NRZ-DPSK clock recovery using an optical resonator. $R T$ : rise time.

Fig. 1 shows the constellation diagram of a $40 \mathrm{Gbit} / \mathrm{s}$ phase modulated signal, as well as the electrical spectrum obtained after detection following filtering with a FP filter having an FSR equal to $40 \mathrm{GHz}$ and a finesse of $F=500$. In the constellation diagram, samples are gathered in a time interval of $0.5 \mathrm{ps}$ around the centre of the bit slot. When the driving signal is not bandwidth limited ( 0 ps rise time), an ideal binary constellation and no tone in the radio frequency (RF) spectrum are observed. On the other hand, when a rise time of $15 \mathrm{ps}$ is introduced, the asymmetry in the distribution of the samples in the constellation diagram is responsible for the appearance of clock tones in the optical spectrum, hence in the RF spectrum obtained after filtering and detection. It should be noted that it is the transfer of voltage fluctuations to optical phase fluctuations by the linear PM that is responsible for the appearance of clock tones. The existence of clock tones in the optical spectrum of bandwidth limited NRZ on-off keying signals has actually already been exploited for their clock recovery at $40 \mathrm{Gbit} / \mathrm{s}$ [4]. Fig. 2 presents eye diagrams of the optical clock obtained at the output of the FP filter for electrical signal rise times of 5, 10 and $20 \mathrm{ps}$ and for a pseudo-random binary sequence (PRBS) length of $2^{7}-1$. All simulations are performed at $40 \mathrm{Gbit} / \mathrm{s}$. Both the clock duty cycle and extinction ratio are dependent on the rise time. Some amplitude fluctuations are visible in the clock signal, as is 
customarily the case when using a FP resonator. However, those can be suppressed using a saturating element such as a semiconductor optical amplifier [1].

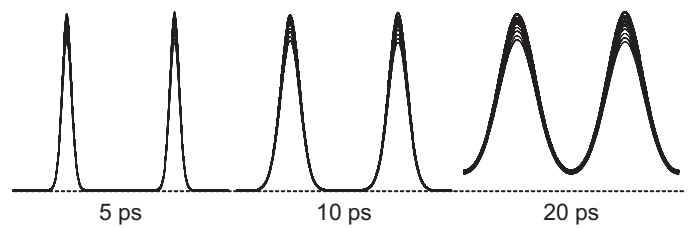

Fig. 2: Optical clock signals at the output of a FP filter with finesse equal to 500 and rise times of 5, 10 and 20 ps. The dotted line indicates the zero power level.

\section{Clock characterisation}

The quality of the recovered clock was analysed as a function of the driving signal rise time for PRBS lengths of $2^{7}-1$ and $2^{11}-1$ and for FP finesses ranging from 100 to 1000. The results are shown in Fig. 3. An average optical power of $0 \mathrm{dBm}$ and a photodiode responsivity of $1 \mathrm{~A} / \mathrm{W}$ are assumed in the calculations.
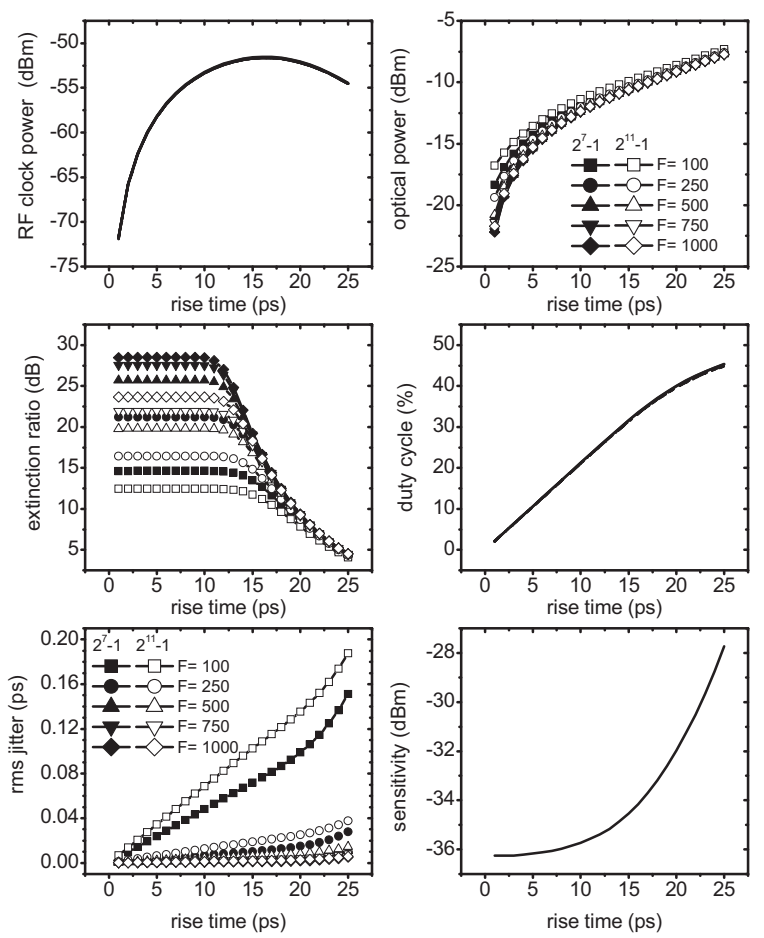

Fig. 3: Characteristics of the retrieved clock signal as a function of the driving signal rise time and the FP filter finesse for PRBS lengths of $2^{7}-1$ and $2^{11}-1$.

The RF clock power and optical clock duty cycle are nearly independent from the finesse and PRBS length. A clear optimum for the RF clock power can be observed around $15 \mathrm{ps}$ rise time. Our calculations indicate that the optimum RF power is at the same level as that obtained directly from the detection of an NRZ-DPSK signal generated by a Mach-Zehnder modulator, which was shown in [5] to be sufficient for generating an optical clock signal with a self-pulsating laser. The optical clock extinction ratio is fairly constant over a broad rise time range, before it starts degrading significantly. As expected, the quality of the recovered optical clock degrades with increasing pattern length, which is typical of such a resonator-based scheme. Nevertheless, a finesse of 250 already guarantees a clock extinction ratio above $15 \mathrm{~dB}$, even for the $2^{11}-1$ pattern. Increasing the finesse to 500 furthermore decreases the deterministic contributions to both the amplitude jitter and the rms timing jitter of the optical clock. Consequently a rise time of $10 \mathrm{ps}$ and $F=500$ are found to be sufficient. Such a rise time introduces only a negligible power penalty on the performance of the NRZ-DPSK signal.
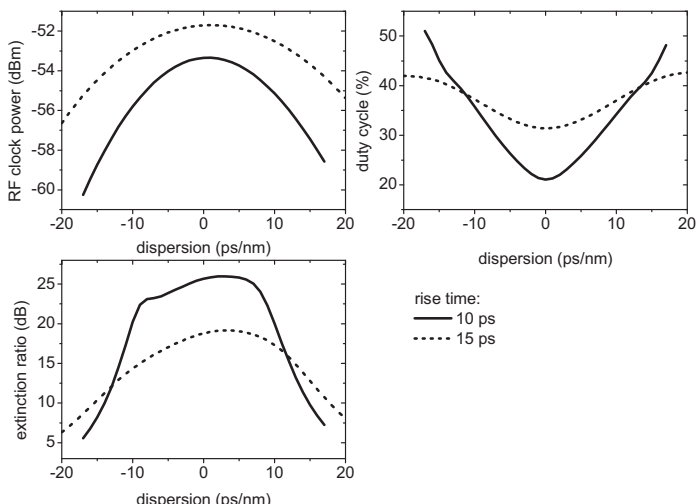

rise time: $10 \mathrm{ps}$
$\cdots \cdots$
$\cdots$

Fig. 4: Dispersion tolerance of the clock recovery scheme for $F=500$ and PRBS length $2^{7}-1$.

The dispersion tolerance of the scheme is shown in Fig. 4 for a FP with $F=500$. In case a 10 ps rise time is used, an optical clock with more than $15 \mathrm{~dB}$ extinction ratio can be reached for a $24 \mathrm{ps} / \mathrm{nm}$ dispersion range. This dispersion tolerance is relatively small. However it corresponds to a compensation ratio range of $99-101 \%$, which is well within the target at $40 \mathrm{Gbit} / \mathrm{s}$ for a standard $80 \mathrm{~km}$ span made of standard single mode fibre and dispersion compensating fibre. With the same optical power, clock enhancement schemes relying on dispersion such as in [3] would result in a maximum RF clock power of $-38 \mathrm{dBm}$ for $40 \mathrm{ps} / \mathrm{nm}$ introduced dispersion. However, such a signal is not directly usable as an optical clock and requires a further optical pulse generation scheme such as a mode-locked laser, unlike the present scheme.

\section{Conclusions}

The clock component that appears in the spectrum of NRZ-DPSK signals for realistic rise time values of the driving signal can be used for all-optical clock recovery using optical resonator filters. The concept was thoroughly characterised using numerical simulations at a bit rate of $40 \mathrm{Gbit} / \mathrm{s}$.

\section{References}

1. G. Contestabile et al., IEEE Photon. Technol. Lett., 18 (2006) 2544.

2. Y. Yu et al., IEEE Photon. Technol. Lett., 18 (2006) 2356.

3. S. Fu et al., IEEE Photon. Technol. Lett., 19 (2007) 925.

4. C. Kim et al., Electron. Lett. 39 (2003) 1456.

5. X. Tang et al., IEEE Photon. Technol. Lett., 20 (2008) 1443. 